Original Paper http://ajol.info/index.php/ijbcs http://indexmedicus.afro.who.int

\title{
Caractérisation physico-chimique et toxicologique des effluents des Centres Hospitaliers et Universitaires du département du Littoral du Bénin
}

\author{
Judicaël Nounagnon TODEDJI ${ }^{1}$, Comlan Cyriaque DEGBEY ${ }^{1,2^{*}}$, Evelyne SOCLO ${ }^{1}$, \\ Arouna YESSOUFOU ${ }^{3}$, Ferdinand GOUDJO $^{4}$, Jean Wilfried HOUNFODJI ${ }^{5}$, \\ Fidele SUANON ${ }^{3}$ et Daouda MAMA ${ }^{3}$
}

\author{
${ }^{1}$ Institut Régional de Santé Publique, Université d'Abomey-Calavi, Ouidah, Bénin. \\ ${ }^{2}$ Clinique Universitaire d'Hygiène Hospitalière, Centre National Hospitalier Universitaire Hubert Koutoukou \\ MAGA, Cotonou, Bénin. \\ ${ }^{3}$ Laboratoire d'Hydrologie Appliquée, Université d'Abomey-Calavi, Bénin. \\ ${ }^{4}$ Laboratoire de Chimie Inorganique et Environnement, Faculté des Sciences et Techniques, Université \\ d'Abomey-Calavi, Bénin. \\ ${ }^{5}$ Laboratoire de Chimie Théorique et de Spectroscopie Moléculaire, Faculté des Sciences et Techniques, \\ Université d'Abomey-Calavi, Bénin. \\ *Auteur correspondant ; E-mail : comlancy@yahoo.fr ; Tél. :0022995960541
}

\section{REMERCIEMENTS}

Ce travail a été financé par l'Université d'Abomey-Calavi à travers le Programme Fonds Compétitifs de Recherche Troisième phase [No 632-2018/UAC/SG/AC/SAF/VR-RU/SPRSP/SA].

\section{RESUME}

Les effluents générés par les activités hospitalières présentent un danger potentiel pour la santé et l'environnement. L'objectif de cette étude était d'étudier la qualité physico-chimique et toxicologique des effluents produits par les Centres Hospitaliers et Universitaires du département du Littoral. Il s'agissait d'une étude transversale descriptive et analytique sur 60 prélèvements d'effluents hospitaliers provenant des stations d'épuration et des fosses septiques. Les échantillons ont été prélevés dans des bouteilles en plastique de 1,5 litre. Les valeurs moyennes des paramètres avec leurs déviations standards ont été calculées. Une analyse en composante principale a été réalisée. Les paramètres physico-chimiques mesurés et comparés aux normes de rejets des polluants dans l'environnement montraient qu'en dehors du $\mathrm{pH}$, de la température et de la conductivité qui étaient conformes aux normes, tous les autres paramètres présentaient des valeurs supérieures aux normes de rejet. Le potentiel hydrogène, la température et la conductivité mesurés sont respectivement en moyenne de 7,14 ; 24,52 ${ }^{\circ} \mathrm{C}$ et $1243,37 \mu \mathrm{S} / \mathrm{Cm}$. Les teneurs moyennes en oxygène dissous, en ammonium et en phosphore sont respectivement de $1,29 \mathrm{mg} / \mathrm{L}, 47,21 \mathrm{mg} / \mathrm{L}$ et $33,18 \mathrm{mg} / \mathrm{L}$. La demande biochimique en oxygène est de 86,33 $\mathrm{mgO}_{2} / \mathrm{L}$ et la demande chimique en oxygène de $164,54 \mathrm{mgO}_{2} / \mathrm{L}$. Les concentrations moyennes du plomb et du cadmium étaient respectivement de $0,53 \mathrm{mg} / \mathrm{L}$ et $0,08 \mathrm{mg} / \mathrm{L}$. Ces effluents présentent donc des risques sanitaires et environnementaux. Il apparait alors plus que nécessaire de développer des méthodes de traitement de ces effluents avant leur rejet dans le milieu naturel.

(C) 2020 International Formulae Group. All rights reserved.

Mots clés : Effluents hospitaliers, qualité chimique, risques sanitaires et environnementaux, Bénin. 


\title{
Physicochemical and toxicological characterization of the effluents of the Hospital and University Centers of the Littoral Department of Benin
}

\begin{abstract}
Effluents generated by hospital activities present a potential danger to health and the environment. The objective was to study the physico-chemical and toxicological quality of the effluents produced by the Hospital and University Centers of the Littoral Department. It was a descriptive and analytical cross-sectional study of 60 samples of hospital effluents from sewage treatment plants and septic tanks. Samples were taken from 1.5 liter plastic bottles. The average values of the parameters with their standard deviations were calculated. A principal component analysis was performed. The physicochemical parameters measured and compared to environmental pollutant release standards showed that, except for the $\mathrm{pH}$, temperature and conductivity that were in compliance with the standards, all other parameters had values that exceeded rejection. The hydrogen potential, the temperature and the conductivity measured are, on average, $7.14,24.52{ }^{\circ} \mathrm{C}$ and $1243.37 \mu \mathrm{S} / \mathrm{cm}$. The average dissolved oxygen, ammonium and phosphorus contents are respectively $1.29 \mathrm{mg} / \mathrm{L}, 47.21 \mathrm{mg} / \mathrm{L}$ and $33.18 \mathrm{mg} / \mathrm{L}$. The biochemical oxygen demand is $86.33 \mathrm{mgO}_{2} / \mathrm{L}$ and the chemical oxygen demand is $164.54 \mathrm{mgO}_{2} / \mathrm{L}$. Mean concentrations of lead and cadmium were $0.53 \mathrm{mg} / \mathrm{L}$ and $0.08 \mathrm{mg} / \mathrm{L}$, respectively. These effluents therefore present health and environmental risks. It appears then more than necessary to develop methods of treating these effluents before their release into the natural environment.
\end{abstract}

(C) 2020 International Formulae Group. All rights reserved.

Keywords: Hospital effluents, chemical quality, health and environmental risks, Benin.

\section{INTRODUCTION}

L'eau tient une place importante dans nos vies en se retrouvant dans toutes les activités qui rythment notre quotidien. Cependant, ses différents usages pour les besoins tels que ceux domestiques, industriels, artisanaux, agricoles constituent des sources de production d'eaux usées qui peuvent contenir de nombreuses substances ainsi que de multiples microorganismes pathogènes présentant des risques pour la santé humaine et menaçant l'environnement. Étant donné que la demande d'eau dans son ensemble augmente, la quantité d'eaux usées produites, et leur charge polluante globale, sont en augmentation constante de par le monde entier (Nations Unies, 2017). Les eaux usées désignent toute eau dont la qualité a été compromise par les activités humaines. Les eaux usées hospitalières peuvent contenir des substances chimiques dangereuses, des agents pathogènes et radio-isotopes pouvant constituer un risque chimique, biologique et physique pour la santé publique et l'environnement (Fekadu et al., 2015). Par rapport aux eaux usées urbaines, les eaux usées des hôpitaux contiennent de diverses substances toxiques ou persistantes telles que les produits pharmaceutiques, les radionucléides, les solvants et les désinfectants à usage médical dans une large gamme de concentrations (Chonova et al., 2016 ; Santos et al., 2013 ; Verlicchi et al., 2012). Verlicchi et al. (2010) ont souligné que les concentrations de micropolluants (par exemple, antibiotiques, analgésiques, métaux lourds) dans les eaux usées des hôpitaux sont entre 4 et 150 fois plus élevées que celles des eaux usées urbaines (Maheshwari et al., 2016).

Malgré les risques liés aux effluents hospitaliers, très souvent, il n'y a pas d'exigences légales en matière de traitement des effluents hospitaliers avant leur rejet dans le collecteur municipal ou directement dans les eaux de surface après le prétraitement. En effet, dans la majorité des pays, il est impossible de trouver des règlements concernant la gestion des effluents hospitaliers et même pas de références spécifiques dans des règlements faisant référence à la gestion des eaux usées en général. Les seules directives existantes concernant les effluents hospitaliers sont celles de l'Organisation Mondiale de la Santé (OMS) en 1999: "Gestion sans danger des déchets d'activités de soins» (WHO, 1999) et mis à 
jour en 2014 (WHO, 2014). La gestion des eaux usées hospitalières pourrait représenter un risque principalement dans les pays en développement, où la majeure partie des eaux usées des soins de santé, sans traitement préalable ou partiel, sont rejetées dans les cours d'eau de surface ou dans les aquifères souterrains par lessivage. Par exemple, les nitrates dans les eaux souterraines des eaux usées non traitées peuvent entraîner une méthémoglobinémie, en particulier chez les bébés. A travers, l'évacuation des eaux usées non traitées dans l'environnement, le nutriment peut entrainer la prolifération d'algues qui favoriseront la production des bactéries potentiellement dangereuses par exemple, des cyanobactéries. Les eaux usées rejetées de manière incontrôlée dans l'environnement peuvent entraîner plusieurs maladies d'origine hydrique qui menacent la vie humaine, en particulier dans les pays en développement.

La caractérisation physico-chimique des effluents hospitaliers comprend l'évaluation de différents paramètres. Parmi ces paramètres, les plus couramment utilisés pour évaluer la présence et les charges de matières inorganiques/organiques dans l'effluent sont la conductivité électrique (CE), la demande biochimique en oxygène (DBO), la demande chimique en oxygène (DCO), le total des solides en suspension (TSS) et l'azote total (El-Ogri et al., 2016). Verlicchi et al (2010) indiquent que les effluents hospitaliers présentent des valeurs de $\mathrm{DBO}_{5}, \mathrm{DCO}$ et TSS généralement 2 à 3 fois plus élevées que celles des effluents municipaux.

Les métaux lourds et éléments à l'état de trace contenus dans les effluents hospitaliers pourraient donner lieu à des dommages pour la santé de l'homme, ainsi que dans les milieux naturels variés (les sols, les eaux et l'air). Les principaux métaux lourds présents dans les effluents hospitaliers sont le gadolinium (Gd), le mercure $(\mathrm{Hg})$ et le platine $(\mathrm{Pt})$ (Verlicchi et al., 2010). D'autres métaux habituellement cherché pour caractériser les effluents hospitaliers, à savoir le cadmium $(\mathrm{Cd})$, l'arsenic $(\mathrm{As})$, le plomb $(\mathrm{Pb})$, le cuivre $(\mathrm{Cu})$, le nickel $(\mathrm{Ni})$, le zinc $(\mathrm{Zn})$ et de chrome $(\mathrm{Cr})$ présentent généralement des concentrations similaires à celles signalées dans les effluents des municipalités (Evens, 2004).

Au Bénin, il n'existe pas de directive spécifique concernant la gestion des effluents hospitaliers. Cependant, le décret $\mathrm{n}^{\circ}$ 2001-109 du 04 avril 2001 fixant les normes de qualité des eaux résiduaires en République du Bénin, fixe les normes de qualité des eaux résiduaires en application des dispositions de la Loi ${ }^{\circ} 98$ 030 du 12 février 1999 portant Loi-cadre sur l'environnement au Bénin. L'objectif de cette étude était d'une part de faire une caractérisation physico-chimique et toxicologique des effluents des Centres Hospitaliers Universitaires du département du Littoral et d'autre part, d'alerter l'opinion publique sur les conséquences potentielles de ces rejets sur l'environnement et les êtres vivants.

\section{MATERIEL ET METHODES \\ Cadre d'étude}

Notre étude avait pour cadre les Centres Hospitaliers Universitaires (CHU) du département du Littoral. Le choix des centres hospitaliers universitaires a été motivé d'une part par le fait qu'ils constituent des centres de référence pour les patients, de formation et d'apprentissage pour les professionnels de la santé et d'autre part par le fait qu'ils produisent beaucoup de déchets liquides dans lesquels on pourra retrouver une gamme importante de polluants. Il existe six centres hospitaliers universitaires dans le département du littoral. Pour des fins de cette recherche, nous avions travaillé dans quatre $\mathrm{CHU}$ sur les six que comporte le département du Littoral: Le Centre National Hospitalier Universitaire Hubert Koutoukou MAGA (CNHU-HKM), le Centre Hospitalier Universitaire de la Mère et Enfant Lagune (CHU-MEL), l'Hôpital d'Instructions des Armées et Centre Hospitalier Universitaire de Cotonou (HIA$\mathrm{CHU}$ ) et le Centre Hospitalier Universitaire de Zone Suru Léré (CHUZ-SL). Ces quatre centres ont été choisis d'une part en raison du fait que les activités menées dans ces centres recouvrent des disciplines, des services médicaux et médico-techniques variés et d'autre part en raison du fait qu'ils offrent une 
diversité de prise en charge de pathologies. Par contre, les deux autres centres sont des centres hospitaliers de prise en charge des pathologies spécifiques. Le réseau de collecte des eaux usées du CNHU-HKM est conçu comme un réseau séparatif dont le fonctionnement n'est pas correct en raison de l'absence d'un réseau d'assainissement pluvial. Le CNHU-HKM dispose d'une station d'épuration à boue activée. Le CHU-MEL dispose d'une station de traitement des eaux usées et utilise également le système des fosses septiques. La station de traitement reçoit une partie des eaux usées produites par les services. Le CHUZ-SL et l'HIA-CHU ne disposent pas de station de traitement et les eaux usées produites sont déversées dans les lavabos ou vidoirs et drainées vers les fosses septiques.

\section{Type d'étude et échantillonnage}

Il s'agissait d'une étude transversale à visée descriptive et analytique sur 60 prélèvements d'effluents bruts hospitaliers provenant des stations d'épuration et des fosses septiques. La méthode non probabiliste avec la technique de choix raisonné a été utilisée pour le choix des fosses septiques des services à prélever. Les effluents des services prélevés étaient ceux qui administraient les soins et qui produisaient plus de déchets liquides présentant en conséquence un risque infectieux plus important. Le CNHU-HKM n'utilisait pas le système de fosses septiques. Les prélèvements d'effluents au niveau des fosses septiques du CHU-MEL, du CHUZ-SL et de l'HIA-CHU étaient respectivement de 17, 20 et 19. Le nombre de prélèvement dans chaque CHU a été déterminé sur la base du nombre de fosses septiques qui reçoivent les effluents des services de soins. Quatre prélèvements ont été réalisés au niveau des stations d'épuration soit deux (2) au niveau CNHU-HKM et deux (2) au CHU-MEL.

\section{Matériel et modalités des prélèvements}

Les échantillons pour l'analyse physicochimique et toxicologique ont été prélevés dans des bouteilles en plastique de 1,5 litre rincées trois fois avec l'effluent à échantillonner. Au niveau des stations d'épuration, des échantillons moyens 24 h ont été collectés. La fréquence des prélèvements a été journalière à des heures précises en fonction des activités de l'hôpital : le matin à 7 heures, l'après-midi à 13 heures et le soir à 19 heures. Ces prélèvements ont été effectués à l'entrée (avant traitement) et à la sortie (après traitement) de la station pour apprécier à chaque niveau la qualité de l'effluent. Après collecte, les échantillons de la journée ont été réunis en un échantillon composite. Au total, quatre échantillons composites ont été constitués. Au niveau des fosses septiques, ces effluents ont été prélevés directement dans les fosses septiques en fin de journée à $19 \mathrm{~h}$.

\section{Conditions de prélèvement et transport des échantillons}

Pour assurer la traçabilité du prélèvement, à chaque point de prélèvement est associée une fiche de prélèvement. Le conditionnement des échantillons pendant le transport est une étape importante qui peut altérer l'intégrité des prélèvements. Afin d'éviter que les échantillons subissent des transformations biologiques, physiques voire chimiques, chaque flacon a été transporté dans des glacières munies des accumulateurs congelés à une température comprise entre $2{ }^{\circ} \mathrm{C}$ et $8{ }^{\circ} \mathrm{C}$ pour refroidir les échantillons mais aussi pour les conserver à l'abri de la lumière. Les échantillons ont été analysés sous $48 \mathrm{~h}$ après le début du prélèvement.

\section{Traitement et analyse des données}

Elle avait comporté deux parties : une première partie qui était consacrée aux analyses en laboratoire et une deuxième aux analyses statistiques. Les analyses en laboratoire ont été effectuées au niveau du Laboratoire de Recherche et d'Expertise en Santé Publique de la Clinique Universitaire d'Hygiène Hospitalière du CNHU-HKM de Cotonou.

\section{Analyse en laboratoire \\ Analyse physico-chimique}

Afin de déterminer l'état de qualité physico-chimique des effluents des $\mathrm{CHU}$, nous nous sommes concentrés sur les paramètres 
suivants: température, potentiel hydrogène, conductivité électrique, total des sels dissous (TDS), turbidité, salinité, demande biochimique en oxygène après 5 jours $\left(\mathrm{DBO}_{5}\right)$, demande chimique en oxygène (DCO), matières en suspension (MES), oxygène dissous, saturation en oxygène, ammonium et phosphores totaux. Ils ont été analysés selon les protocoles recommandés par Rodier et al. (2009). Les méthodes d'analyses utilisées sont présentées dans le Tableau 1 avec les paramètres mesurés.

\section{Analyse toxicologique : Analyse des métaux}

L'analyse des métaux lourds a concerné le dosage du Plomb et du Cadmium. Les métaux lourds ont été analysés au spectrophotomètre d'absorption atomique à flamme par la méthode spectrométrique à la
Dithizone après minéralisation des échantillons.

\section{Analyse statistique des données}

Les différentes analyses statistiques ont été réalisées avec les logiciels SPSS, version 21 et OriginPro 9.0. Les concentrations moyennes des paramètres et leur écart type ont été calculées et comparées aux valeurs guides de l'Organisation Mondiale de Santé (2014) et celles du Bénin. A partir de l'analyse en composantes principales ( $\mathrm{ACP}$ ), la réalisation de la carte factorielle, la corrélation entre les différents paramètres et la classification des points de prélèvement en fonction des paramètres physico-chimiques ont été établies au seuil de signification $\alpha=0,05$.

Tableau 1: Méthodologie d'analyse des paramètres physico-chimiques et toxicologiques.

\begin{tabular}{|c|c|c|}
\hline Paramètres & Matériels & Méthodes utilisées \\
\hline Température & Multi $3320 \mathrm{WTW}$ & Mesure directe par le Multi 3320 WTW \\
\hline Potentiel hydrogène & Multi 3320 WTW & Mesure directe par le Multi 3320 WTW \\
\hline Conductivité électrique & Multi 3320 WTW & Mesure directe par le Multi 3320 WTW \\
\hline Turbidité & Multi 3320 WTW & Mesure directe par le Multi 3320 WTW \\
\hline Salinité & Multi 3320 WTW & Mesure directe par le Multi 3320 WTW \\
\hline Total des sels Dissous & Multi 3320 WTW & Mesure directe par le Multi 3320 WTW \\
\hline Oxygène dissous & Multi 3320 WTW & Mesure directe par le Multi 3320 WTW \\
\hline Saturation en oxygène & Multi 3320 WTW & Mesure directe par le Multi 3320 WTW \\
\hline Ammonium & Spectrophotomètre & $\begin{array}{l}\text { Méthode spectrophotométrique au réactif } \\
\text { de Nessler }\end{array}$ \\
\hline Phosphores totaux & Spectrophotomètre & Méthode spectrophotométrique \\
\hline $\mathrm{DBO}_{5}$ & Oxytope Respiromètre & Manométrique \\
\hline DCO & Colorimètre & Procédé de dichromate de potassium \\
\hline MES & Centrifugeuse & Méthode par centrifugation \\
\hline Plomb & $\begin{array}{l}\text { Spectrophotomètre d'absorption } \\
\text { atomique à flamme }\end{array}$ & Méthode spectrométrique à la dithizone \\
\hline Cadmium & $\begin{array}{l}\text { Spectrophotomètre d'absorption } \\
\text { atomique à flamme }\end{array}$ & Méthode spectrométrique à la dithizone \\
\hline
\end{tabular}




\section{RESULTATS}

\section{Caractérisation physico-chimique des effluents hospitaliers}

Les moyennes des concentrations des paramètres physico-chimiques des quatre $\mathrm{CHU}$ par rapport à certaines normes sont présentées dans le Tableau 2. Les résultats des analyses ont été comparés à la norme de l'OMS et à celles adoptées au Bénin. En dehors du pH, de la température et de la conductivité qui étaient conformes aux normes, tous les autres paramètres présentent des valeurs supérieures aux normes de rejet des eaux usées dans l'environnement.

\section{Caractérisation toxicologique des effluents hospitaliers}

Les moyennes des concentrations en métaux lourds des quatre CHU sont présentées dans le Tableau 3. Les concentrations du plomb et du cadmium varient d'un CHU à un autre avec une concentration moyenne de $0,53 \mathrm{mg} / \mathrm{L}$ pour le plomb et $0,08 \mathrm{mg} / \mathrm{L}$ pour le cadmium. L'HIA-CHU présente une valeur moyenne en cadmium très élevée par rapport à la norme $(0,13 \mathrm{mg} / \mathrm{L})$.

\section{Analyse en composante des paramètres physico-chimiques sur l'ensemble des échantillons}

L'analyse en composante principale réalisée montre que $63,44 \%$ de la variance totale a été expliqué; avec $36,84 \%$ comptant pour l'axe factoriel 1 (F1) et $26,60 \%$ pour l'axe factoriel 2 (F2) (Figure 1). De l'analyse de la figure, dans le plan formé par les axes factoriels 1 et 2 (F1 et F2), les variables les plus corrélées à l'axe F1 sont: conductivité électrique, total des sels dissous, turbidité, salinité, température, matières en suspension, saturation en oxygène, oxygène dissous. Parmi ces variables, celles qui contribuent le plus à former l'axe F1 se répartissent en deux groupes différents: le premier regroupe conductivité électrique, matières en Suspension, salinité, turbidité, total des sels dissous et température, qui sont fortement corrélés entre elles et positivement à l'axe $\mathrm{F} 1$, puisqu'elles définissent des vecteurs propres de même direction et le deuxième groupe qui est rassemblé par la saturation en oxygène et l'oxygène dissous, qui sont corrélées négativement à l'axe F1. Les corrélations entre ces variables sont d'autant plus fortes lorsque les variables sont positionnées aux extrémités de l'axe défini par l'axe F1. Cela met en évidence la possibilité que ces polluants proviennent de la même source. Sur l'axe 2, les variables les plus corrélées à F2 sont : DCO, $\mathrm{DBO}_{5}$ et $\mathrm{pH}$. D'autres variables (ammonium et phosphores Totaux) étant proches du centre du plan factoriel, leur corrélation n'est certainement pas très forte. Ces variables sont probablement mieux expliquées par d'autres composantes principales, autres que les F1 et F2. Afin d'identifier les liens existants entre les échantillons et leur composition chimique, les coordonnées des observations dans le plan factoriel F1 x F2 ont été représentées sur la Figure 1. La carte factorielle des échantillons confirme que la qualité physico-chimique des effluents hospitaliers varie d'un site de prélèvement à un autre. Les effluents prélevés au niveau des sites encadrés en bleu sont fortement pollués par les matières organiques car ils ont des valeurs fortement élevées de $\mathrm{DBO}_{5}$ et DCO. Les sites $\mathrm{P} 39$ à $\mathrm{P} 41$ sont représentés par les fosses septiques du service des urgences et P33-34, les services de médecine de l'HIA-CHU et le P1 représente les effluents en entrée de la station du CNHUHKM. Les sites de prélèvement encadrés en rouge présentes des valeurs de TDS, salinité et de CE supérieures aux normes de rejet des eaux usées dans l'environnement. Ils sont les plus riches en différentes formes organiques et inorganiques de pollution. Ceux encadrés en vert présentent des valeurs de l'oxygène dissous et de saturation en oxygène élevées 


\section{Corrélations entre variables}

L'étude des corrélations linéaires bivariées entre les paramètres étudiés renseigne que des corrélations intéressantes sont observées. La matrice des corrélations des paramètres physico-chimiques mesurés durant notre étude a été présentée dans le Tableau 4. Dans ce tableau où les coefficients de corrélation de Pearson significatifs $(\mathrm{p}<0,05)$ sont représentés en gras, des corrélations intéressantes sont observées. Des corrélations fortes et positives ont été observées d'une part entre la conductivité électrique et le TDS $(0,878)$ ainsi qu'avec la salinité $(0,978)$ et d'autre part entre la turbidité et les matières en suspension $(0,701)$ ainsi qu'avec la $\mathrm{DBO}_{5}$ $(0,836)$ et la DCO $(0,829)$. Une forte corrélation et positive existe entre la $\mathrm{DBO}_{5}$ et la DCO $(0,988)$. Pa ailleurs une corrélation négative a été observée entre la turbidité et la saturation en oxygène $(-0,502)$. Il a été observé également une forte corrélation et positive entre l'ammonium et le les phosphores totaux $(0,943)$.

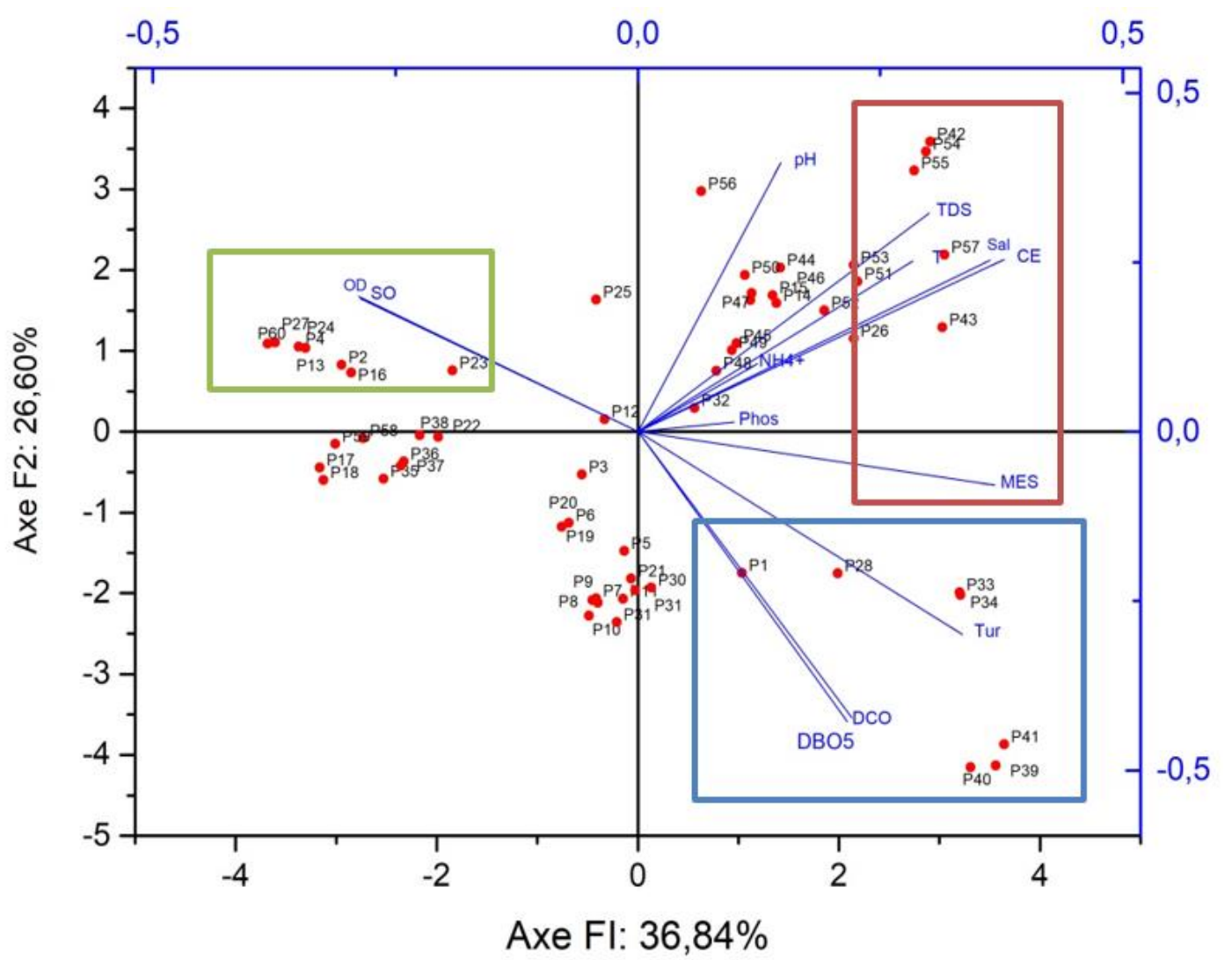

T : Température, CE : Conductivité, Tur :Turbidité, Sal : Salinité, TDS : Total des Sels Dissous, OD : Oxygène dissous, SO : Saturation en oxygène, NH4+ : Ammonium, Phos : Phosphores totaux : DBO5 :Demande biochimique en oxygène, DCO : Demande chimique en oxygène, MES : Matières en suspension.

P1 à P60 : Différents points de prélèvement.

Figure 1 : Approche graphique de l'ACP des paramètres physico-chimiques dans les effluents et projection des points de prélèvement dans l'espace des axes F1 et F2. 
Tableau 2 : Caractéristiques physico-chimiques des effluents des CHU.

\begin{tabular}{|c|c|c|c|c|c|c|c|}
\hline Paramètres & Unités & CNHU-HKM & CHU-MEL & HIA-CHU & CHUZ-SL & Moyenne & $\begin{array}{l}\text { Normes } \\
\text { Bénin/OMS }\end{array}$ \\
\hline Température & ${ }^{\circ} \mathrm{C}$ & $22,75 \pm 0,21$ & $21,44 \pm 1,11$ & $24,97 \pm 1,71$ & $27,29 \pm 2,98$ & $24,52 \pm 3,12$ & $<30$ \\
\hline $\mathrm{pH}$ & - & $7,06 \pm 0,02$ & $7,00 \pm 0,47$ & $6,94 \pm 0,47$ & $7,49 \pm 0,48$ & $7,14 \pm 0,52$ & $6-9$ \\
\hline Conductivité & $\mathrm{S} / \mathrm{cm}$ & $974,5 \pm 354,26$ & $844,0 \pm 556,08$ & $945,75 \pm 635,10$ & $1984,32 \pm 943,04$ & $1243,37 \pm 870,81$ & $<2000$ \\
\hline Turbidité & NTU & $81,85 \pm 61,59$ & $68,40 \pm 34,69$ & $81,26 \pm 71,17$ & $63,91 \pm 35,88$ & $71,71 \pm 50,13$ & $<5$ \\
\hline Salinité & $\%$ & $0,45 \pm 0,21$ & $0,36 \pm 0,32$ & $0,45 \pm 0,41$ & $1,04 \pm 0,58$ & $0,61 \pm 0,53$ & 0 \\
\hline TDS & $\mathrm{mg} / \mathrm{L}$ & $487,50 \pm 177,48$ & $540,95 \pm 619,69$ & $479,50 \pm 315,94$ & $1761,26 \pm 849,87$ & $905,12 \pm 846,32$ & \\
\hline $\begin{array}{l}\text { Oxygène } \\
\text { dissous }\end{array}$ & $\mathrm{mg} / \mathrm{L}$ & $2,10 \pm 2,40$ & $1,26 \pm 1,40$ & $1,13 \pm 1,15$ & $1,40 \pm 0,90$ & $1,29 \pm 1,18$ & $>5$ \\
\hline $\begin{array}{l}\text { Saturation en } \\
\text { oxygène }\end{array}$ & $\%$ & $23,50 \pm 26,16$ & $14,37 \pm 15,88$ & $13,13 \pm 13,25$ & $15,93 \pm 10,35$ & $14,76 \pm 13,48$ & - \\
\hline Ammonium & $\mathrm{mg} / \mathrm{L}$ & $5,73 \pm 7,96$ & $45,67 \pm 105,61$ & $58,96 \pm 132,41$ & $40,73 \pm 58,09$ & $47,21 \pm 100,99$ & $<0,2$ \\
\hline $\begin{array}{l}\text { Phosphores } \\
\text { totaux }\end{array}$ & $\mathrm{mg} / \mathrm{L}$ & $15,03 \pm 4,05$ & $39,64 \pm 44,27$ & $41,32 \pm 69,25$ & $20,05 \pm 22,31$ & $33,18 \pm 48,96$ & $<10$ \\
\hline $\mathrm{DBO}_{5}$ & $\mathrm{mgO}_{2} / \mathrm{L}$ & $190,00 \pm 0,00$ & $85,46 \pm 71,62$ & $145,19 \pm 176,26$ & $23,38 \pm 10,47$ & $89,00 \pm 120,04$ & $<25^{*}$ \\
\hline DCO & $\mathrm{mgO}_{2} / \mathrm{L}$ & $356,69 \pm 0,00$ & $159,22 \pm 132,90$ & $287,67 \pm 333,76$ & $37,23 \pm 15,82$ & $169,99 \pm 230,06$ & $<125^{*}$ \\
\hline MES & $\mathrm{mg} / \mathrm{L}$ & $86,50 \pm 79,90$ & $66,16 \pm 26,90$ & $105,55 \pm 89,47$ & $119,84 \pm 63,29$ & $96,97 \pm 68,06$ & $35^{*}$ \\
\hline
\end{tabular}

$\mathrm{DBO}_{5}$ : Demande biochimique en oxygène, DCO : Demande chimique en oxygène, MES : Matières en suspension, TDS : Total des Sels Dissous. 
Tableau 3 : Valeur moyenne des concentrations en mg/L des métaux lourds des effluents des CHU comparée aux normes l'OMS.

\begin{tabular}{llllllll}
\hline Paramètres & Unités & CNHU-HKM & CHU-MEL & HIA-CHU & CHUZ-SL & Moyenne & Normes OMS \\
\hline Plomb & $\mathrm{mg} / \mathrm{L}$ & $0,38 \pm 0,23$ & $0,69 \pm 0,69$ & $0,21 \pm 0,08$ & $0,73 \pm 1,51$ & $0,53 \pm 0,95$ & 5 \\
Cadmium & $\mathrm{mg} / \mathrm{L}$ & $0,07 \pm 0,00$ & $0,06 \pm 0,04$ & $0,13 \pm 0,09$ & $0,06 \pm 0,03$ & $0,08 \pm 0,07$ & 0,01 \\
\hline
\end{tabular}

Tableau 4: Matrice de corrélation des paramètres physiques et chimiques sur l'ensemble des échantillons.

\begin{tabular}{|c|c|c|c|c|c|c|c|c|c|c|c|c|c|}
\hline & pH & $\mathbf{C E}$ & TDS & Tur & Sal & $\mathbf{T}$ & MES & Oxy & Sat & $\mathrm{DBO}_{5}$ & DCO & NH4+ & P-PO43- \\
\hline $\mathrm{pH}$ & 1 & & & & & & & & & & & & \\
\hline $\mathrm{CE}$ & 0,566 & 1 & & & & & & & & & & & \\
\hline TDS & 0,538 & 0,878* & 1 & & & & & & & & & & \\
\hline Tur & $-0,154$ & 0,363 & 0,15 & 1 & & & & & & & & & \\
\hline Sal & 0,554 & 0,978* & $0,833 *$ & 0,342 & 1 & & & & & & & & \\
\hline $\mathrm{T}$ & 0,479 & 0,688* & $0,609 *$ & 0,199 & $0,664 *$ & 1 & & & & & & & \\
\hline MES & 0,155 & $0,513^{*}$ & 0,456 & 0,701 & 0,444 & 0,532 & 1 & & & & & & \\
\hline Oxy & 0,084 & $-0,292$ & $-0,171$ & $-0,493$ & $-0,266$ & $-0,12$ & $-0,379$ & 1 & & & & & \\
\hline Sat & 0,08 & $-0,288$ & $-0,17$ & $-0,502 *$ & $-0,259$ & $-0,122$ & $-0,392$ & 0,995* & 1 & & & & \\
\hline $\mathrm{DBO}_{5}$ & $-0,347$ & 0,072 & $-0,15$ & $0,836 *$ & 0,073 & $-0,034$ & 0,531 & $-0,378$ & $-0,384$ & 1 & & & \\
\hline $\mathrm{DCO}$ & $-0,367$ & 0,064 & $-0,162$ & $\mathbf{0 , 8 2 9} *$ & 0,065 & $-0,043$ & 0,506 & $-0,388$ & $-0,395$ & 0,988* & 1 & & \\
\hline NH4+ & 0,262 & 0,172 & 0,077 & 0,065 & 0,174 & 0,07 & 0,211 & $-0,22$ & $-0,219$ & $-0,139$ & $-0,148$ & 1 & \\
\hline
\end{tabular}

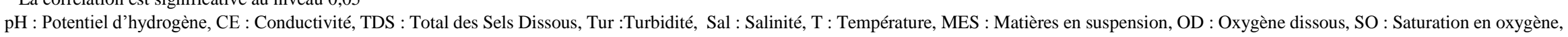
$\mathrm{DBO}_{5}$ :Demande biochimique en oxygène, DCO : Demande chimique en oxygène, NH4+ : Ammonium, Phos : Phosphores totaux. 


\section{DISCUSSION}

Les effluents produits par les hôpitaux présentent une grande variabilité des caractéristiques et cette variabilité dépend de la taille des hôpitaux, du nombre de lit, du nombre de patients hospitalisés et ambulatoires, du nombre et du type des services, du pays et des saisons (Verlicchi et al., 2012 ; Al Aukidy et al., 2014). Cette étude a montré la qualité physico-chimique et toxicologique des effluents produits par les CHU du département du Littoral et les effets inhérents qu'elle peut poser sur l'environnement de réception. Selon les caractéristiques physico-chimiques, les effluents hospitaliers recueillis ont révélé que seuls le $\mathrm{pH}$, la température et la conductivité électrique présentaient des valeurs moyennes conformes aux normes de rejet des eaux usées dans l'environnement. Nos résultats se rapprochent des travaux de la littérature qui ont rapporté également que les rejets liquides du milieu hospitalier sont très chargés en polluants chimiques et germes pathogènes et constituent de ce fait une menace pour l'environnement et la santé (Berrada et al., 2014 ; Ike et al., 2017 ; Sadek et al., 2012; El Mehdi et al., 2008; Bouzid et al., 2013 ; Aissi et al., 2013). Leur traitement préalable devient une nécessité absolue (Berrada et al., 2014). Ainsi, l'étude menée par Ike et al. (2017) au Nigéria a montré que les valeurs des paramètres physicochimiques mesurés sont élevées dans les échantillons des effluents hospitaliers, et toutes les valeurs sont au-dessus des normes réglementaires nigérianes de rejet des eaux usées dans l'environnement, sauf pour la température qui a une valeur en dessous des normes. Les valeurs moyennes de température et de $\mathrm{pH}$ des effluents au niveau des quatre CHU sont conformes aux normes béninoises, en matière de rejet des déchets liquides qui préconisent une température de $30^{\circ} \mathrm{C}$ et un $\mathrm{pH}$ compris entre 6 et 9 pour les eaux usées domestiques. La température joue un rôle très important dans la solubilité des sels, des gaz et la détermination du $\mathrm{pH}$. Il agit également comme un facteur physiologique sur le métabolisme des micro-organismes vivant dans l'eau. Dans notre étude, la température varie de $18,6{ }^{\circ} \mathrm{C}$ à $30,2{ }^{\circ} \mathrm{C}$ avec une moyenne de $24,52{ }^{\circ} \mathrm{C}$, ce qui est conforme à la norme internationale établie par l'OMS (2009) qui fixait la température maximale à $30{ }^{\circ} \mathrm{C}$. La température trouvée par Sadek et al. (2012) dans les effluents du centre hospitalier provincial de Sidi Kacen était de $19,8^{\circ} \mathrm{C}$, celle trouvée par Tahiri et al. (2009) était de 17,11 ${ }^{\circ} \mathrm{C}$. L'étude menée par Bouzid et al. (2013) à l'hôpital Mohamed V de Meknès au Maroc a rapporté une température comprise entre 19,4 ${ }^{\circ} \mathrm{C}$ et $20,4{ }^{\circ} \mathrm{C}$. Les résultats du pH présentés dans cette étude ont montré que ces effluents ont un pH qui oscille entre 6,18 et 8,09 avec une moyenne de 7,14. Ce résultat est conforme à celui de l'OMS $(6,5-8,5)$ et des résultats obtenus dans plusieurs études (Sadek et al., 2012 ; El Mehdi et al., 2008 ; Bouzid et al., 2017). La conductivité électrique est utilisée pour estimer la minéralisation globale et le total des sels solubles dans l'eau et est probablement l'un des plus simples et le plus important indicateur de contrôle de la qualité des effluents hospitaliers (Rodier et al., 2009). Dans notre étude, la conductivité électrique varie de 245 à $3210 \mu \mathrm{S} / \mathrm{cm}$. Malgré une valeur moyenne conforme aux normes, les valeurs de la conductivité des effluents des services de la buanderie de l'Hôpital d'Instruction des Armées et Centre Hospitalier Universitaire et du Centre Hospitalier Universitaire de Zone de Suru Léré et ceux des services des urgences et de la pédiatrie du CHUZ-SL présentent des valeurs supérieures aux normes de rejets dans l'environnement. Les valeurs obtenues dans la majorité des échantillons d'effluents des CHU restent en dessous de la limite inférieure de $2000 \mu \mathrm{S} / \mathrm{cm}$ de l'OMS. Les effluents de ces quatre $\mathrm{CHU}$ ont des valeurs moyennes de conductivité inférieures à celles des effluents des zones sanitaires de Calavi et de Sô-Ava (Aissi et al., 2013). En effet, la valeur de conductivité de ces effluents varie entre 3700 $\mu \mathrm{S} / \mathrm{cm}$ et $4260 \mu \mathrm{S} / \mathrm{cm}$. Plus encore, des valeurs plus élevées de la conductivité $(3140 \mu \mathrm{s} / \mathrm{cm})$ ont été notées à l'hôpital de Sidi Kacem (Sadek et al., 2012). Une eau de conductivité comprise entre 600 et $1000 \mu \mathrm{S} / \mathrm{cm}$ étant considérée 
comme une eau à forte minéralisation (Rodier et al., 2009), les effluents de nos quatre CHU sont des eaux usées fortement minéralisées. La salinité suit les mêmes tendances que la conductivité électrique (Sadek et al., 2012 ; El Morhit et al., 2012). En effet, la valeur de la salinité varie entre 0,1 et $1,8 \mathrm{~g} / \mathrm{l}$, avec une moyenne $0,61 \mathrm{~g} / \mathrm{l}$. Cette moyenne est inférieure à la valeur moyenne de salinité obtenue dans l'étude de El Mehdi et al. (2009). La matière en suspension représente l'ensemble des particules minérales et organiques contenues dans les eaux usées (Rodier et al., 2009). En effet, dans la présente étude, la teneur moyenne en MES $(96,97 \mathrm{mg} / \mathrm{l})$ obtenue dépasse la valeur limite des normes de l'OMS (20 mg/l) pour les rejets des eaux usées. Ces résultats corroborent le faible niveau d'oxygène dissous en ce sens que leur dégradation consomme une quantité importante d'oxygène disponible. Ces résultats sont souvent liés aux matériaux des charges organiques et minéraux engendrés par les différentes activités des centres hospitaliers universitaires. Or, la présence de la MES dans les eaux souterraines présente un risque pour la production d'eau potable, car elles sont porteuses de micro-organismes. Les matières solides en suspension représentent tous les minéraux et les particules organiques contenues dans les eaux usées. La connaissance de la concentration d'éléments colloïdaux dans les eaux usées est nécessaire pour évaluer l'impact de la pollution sur l'environnement aquatique. Les concentrations d'ammonium $\mathrm{NH}_{4}{ }^{+}$du CHU-MEL, de l'HIA-CHU et du CHUZ-SL sont très élevées par rapport à la norme de l'OMS qui est de $0,2 \mathrm{mg} / \mathrm{l}$. La présence d'ammoniac en grandes quantités est indicative de la contamination par les rejets d'origine humaine probablement dues à la transformation de la vitesse de l'urée en ammoniac (EL Mountassir et al., 2017).

\section{Analyse des indicateurs de pollution}

Les résultats ont montré que les effluents étaient très chargés en polluants et constituent une menace pour l'environnement

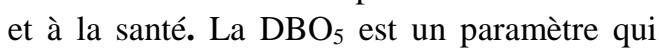
permet d'évaluer la fraction de matières organiques biodégradables. L'analyse des résultats montre que les concentrations en $\mathrm{DBO}_{5}$ sont très élevées, $89,00 \mathrm{mg} / \mathrm{L}$ au lieu de $25 \mathrm{mg} / \mathrm{L}$, comme le préconise la norme des rejets domestiques au Bénin. Selon la norme de l'OMS, la DCO pour les effluents doit être d'au moins $90 \mathrm{mg} / \mathrm{L}$. Dans les effluents des CHU de notre étude, nous avons enregistré une DCO moyenne de 169,99 mg L de $\mathrm{O}_{2}$ contre 125 $\mathrm{mg} / \mathrm{L}$ de $\mathrm{O}_{2}$ pour la norme acceptée au Bénin. Le rapport DCO/DBO5 $(1,91)$ est inférieur à 3 , ce qui montre que les effluents sont biodégradables (Rodier et al., 2009). Ce rapport détermine la possibilité et le rendement de dégradation que l'on peut espérer par un traitement d'oxydation biologique. Cependant, certains travaux ont donné des résultats plus élevés que ceux de notre étude. Des concentrations moyennes plus élevées de la DCO $(820,20 \mathrm{mg} / \mathrm{l})$ ont été obtenues dans les effluents de l'hôpital Al Ghassani en 2009. En ce qui concerne la $\mathrm{DBO}_{5}$, des concentrations moyennes plus élevées $(1881,1 \mathrm{mg} / \mathrm{l})$ ont été enregistrées dans les effluents de l'hôpital Al Ghassani (El Mehdi et al., 2009).

En ce qui concerne les métaux, la composition des produits utilisés, dans les services de soins des quatre $\mathrm{CHU}$, révèle une concentration élevée en cadmium et plomb. L'HIA-CHU présente une valeur moyenne en cadmium très élevée par rapport à la norme $(0,13 \mathrm{mg} / \mathrm{L})$. Les résultats montrent que la concentration en métaux des eaux usées varie au niveau de chaque hôpital. La teneur élevée en cadmium à l'HIA-CHU pourrait être dû aux activités de nettoyage qui emploient de grands volumes de détergents, à l'utilisation des produits de teinture lors de la réfection des salles de soins et à l'absence du prétraitement des effluents au niveau des services avant leur évacuation dans les fosses septiques. La présence des métaux lourds dans les effluents hospitaliers est liée à la présence d'agents de contraste iodés utilisés pour la radiographie, de certains médicaments et de leurs métabolites qui peuvent contenir des éléments organohalogénés, de l'utilisation de désinfectants, de détergents et de solvants chlorés, ainsi que d'autres substances provenant de laboratoires. Les concentrations moyennes de plomb retrouvées dans les 
effluents sont inférieures aux normes béninoises de rejet. Cependant, les activités anthropiques telles que la combustion de combustibles fossiles, l'incinération des ordures ménagères et industrielles pourraient conduire à une augmentation des concentrations des métaux lourds dans les compartiments environnementaux (air, eau, sol). Parmi les différents éléments métalliques émis dans l'environnement lors des activités anthropiques, le zinc, le plomb, le chrome, le cadmium, le cuivre et le nickel sont les plus couramment mesurés dans les études environnementales en raison de la diversité des sources d'émission (Chiffoleau, 2001).

Cependant, bien que ces concentrations soient faibles, l'introduction continuelle de métaux lourds en milieu aquatique est à l'origine d'effets néfastes sur les organismes marins à cause de leurs propriétés de bioaccumulation et de biomagnification (Lim et Schoenung, 2010; Baby et al., 2010). Le cadmium et le plomb sont des toxiques cumulatifs, capables de s'accumuler dans les produits alimentaires aquatiques et d'occasionner un certain nombre de perturbations chez l'homme : atteintes rénales, osseuses, neurologiques et/ou digestives, troubles cancérogènes et/ou mutagènes (Oliveira et al., 2012 ; Prozialeck et al., 2012). Le cadmium est un métal de transition reconnu comme étant cancérogène, mutagène et cytotoxique. Des données récentes suggèrent que les protéines qui participent à des systèmes de réparation de l'ADN, en particulier dans l'excision et la réparation des mésappariements, sont des cibles sensibles de la toxicité du cadmium (Baby et al., 2010).

Dans le plan factoriel F1 x F2, les variables les plus corrélées à l'axe F1 sont: conductivité électrique, total des sels dissous, turbidité, salinité, température, matières en suspension, saturation en oxygène, oxygène dissous. Elles sont corrélées positivement à F1, mais on a aussi les variables saturation en oxygène et oxygène dissous qui sont corrélées négativement à F1. Cet axe peut être assimilé au caractère minéral et organique des effluents dont dépends les paramètres indiqués cidessous. Sur l'axe défini par l'axe 2, les variables les plus corrélées à cette composante principale sont : $\mathrm{DCO} \mathrm{DBO}_{5}$ et $\mathrm{pH}$. Le facteur F2 exprime donc une pollution organique des effluents.

Les groupes de variables encadrées en "rouge" et en "vert" corrélées à l'axe F1 témoignent donc d'une pollution minérale et organique des effluents hospitaliers. Le groupe de variables encadrées en "bleu" et corrélées à l'axe F2 témoigne d'une pollution organique. L'opposition observée entre le groupe de variables encadrées en "vert" corrélées négativement à F1 et celles encadrées en "rouge" corrélées positivement à F1 est dû au faite que la dégradation de la matière organique et minérale contenue dans les effluents hospitaliers consomme une quantité importante d'oxygène disponible dans les effluents. Il en est de même pour l'opposition observée entre les groupes de variables encerclées en "bleu" corrélées à F2 et celles encadrées en "vert". La dégradation de la pollution organique consomme l'oxygène du milieu donc plus la quantité de matière organique augmente et plus on note une diminution de la quantité d'oxygène. Dans la présente étude, les valeurs de la conductivité électrique, de la turbidité, des matières en suspension, de $\mathrm{la}_{\mathrm{DBO}}$ et de la DCO corroborent le faible niveau d'oxygène dissous et de la saturation en oxygène. Hamaidi et al. (2009), affirment que la faible oxygénation de l'eau peut être due soit à une consommation élevée d'oxygène dissous par les bactéries aérobies pour la dégradation de la matière organique, soit à une faible activité photosynthétique du phytoplancton. Il confère aux effluents un caractère anoxique et donc réducteur et asphyxiant. Les résultats de l'ACP réalisée dans l'étude menée par Morhit et al. (2015) ont rapporté que l'axe F1 représenté par température de l'air et de l'eau définit un gradient thermique. L'axe F2 déterminé par la salinité et la conductivité définit un gradient de minéralisation. Ces auteurs affirment que cet axe est caractérisé par la minéralisation significative liée aux activités anthropogènes.

\section{Corrélations entre variables}

L'étude des corrélations linéaires bivariées entre les paramètres étudiés renseigne 
que des corrélations intéressantes sont observées (Tableau 4). Des corrélations fortes et positives ont été observées entre la conductivité électrique et le TDS $(0,878)$ ainsi qu'avec la salinité $(0,978)$. La forte corrélation observée entre ces paramètres s'explique par le fait qu'ils sont des indicateurs de la pollution minérale d'une eau usée. La conductivité électrique est utilisée pour estimer la minéralisation globale et le total des sels solubles dans l'eau (Rodier et al., 2009). La salinité suit les mêmes tendances que la conductivité électrique (El Morhit et al., 2012; Sadik et al., 2012). Une forte corrélation positive a été observée entre la turbidité et les matières en suspension $(0,701)$. La turbidité d'une eau est due à la présence des particules en suspension, notamment colloïdales. L'existence d'une corrélation positive entre la turbidité avec la $\mathrm{DBO}_{5}(0,836)$ et la DCO $(0,829)$ s'explique par le fait qu'ils sont des indicateurs de la pollution organique d'une eau usée. La forte corrélation positive observée entre la $\mathrm{DBO}_{5}$ et la DCO $(0,988)$ se justifie par le fait que les deux paramètres reflètent la contribution significative des matières organiques présentes dans les effluents hospitaliers. Le rapport COD/BOD5 laisse estimer la biodégradabilité de la matière organique, pour déterminer le degré de pollution (Carraro et al., 2016). Par ailleurs une corrélation négative a été observée entre la turbidité et la saturation en oxygène $(-0,502)$ puisque la turbidité d'une eau est due à la présence des particules en suspension or la dégradation de ces particules nécessite une consommation d'oxygène. Il a été observé également une forte corrélation positive entre l'ammonium et le les phosphores totaux $(0,943)$. L'ammonium et le phosphore constituent des éléments nutritifs indispensables aux végétaux et sont des substances eutrophisantes. Des concentrations de nitrates et de phosphates trop importantes induisent le phénomène d'eutrophisation (étouffement de la vie aquatique).

\section{Conclusion}

La plupart des paramètres physicochimiques dépasse les normes admises. Cela montre que ces effluents présentent des risques énormes pour les organismes vivants dans les réserves d'eau naturelles. Ce résultat peut être expliqué par la forte activité médicale qui se mène au niveau de ces CHU et par l'absence du traitement correcte de ces effluents produits par ces centres hospitaliers. Ces eaux usées se caractérisent donc par de fortes charges organiques résultant de la série de pratiques hospitalières. Dans la plupart des cas, les eaux usées de l'hôpital ne sont pas systématiquement traitées avant l'élimination dans l'environnement, posant ainsi des risques sérieux pour la santé et d'autres menaces environnementales parmi les habitants environnants. Il apparait alors plus que nécessaire de développer des méthodes de traitement de ces effluents avant leur rejet dans le milieu naturel.

\section{CONFLIT D'INTERETS}

Tous les auteurs ne déclarent aucun conflit d'intérêts.

\section{CONTRIBUTIONS DES AUTEURS}

JNT est l'investigateur principal et a participé à la planification et à la réalisation de l'étude. Il a effectué la saisie et l'analyse des données. CCD, AY, FS et DM ont participé à la planification de l'étude et ont contribué au processus de rédaction. ES, FG et JWH ont participé à l'analyse des données et ont contribué au processus de rédaction. Tous les auteurs ont lu et approuvé le manuscrit final.

\section{REFERENCES}

Aissi AK, Edorh PA, Degbey C, Guedenon P, Segbo J, Hounkpatin ASY, Boko M, Loko F. 2013. Assessment of risk connected to the management of effluents from Abomey-Calavi and So-Ava Laboratories. British Journal of Applied Science \& Technology, 3(2): 307-319. DOI: http://dx.doi.org/10.9734/BJAST/2014/2 296

Al Aukidy M, Verlicchi P, Voulvoulis N. 2014. A framework for the assessment of the environmental risk posed by pharmaceuticals originating from hoapital effluents. Sci Total Environ, 493: 54-64. 
DOI:

http://dx.doi.org/10.1016/j.scitotenv.2014. 05.128

Baby J, Raj JS, Biby, Etsankarganesh P, Jeevitha MV, Ajisha SU, Rajan SS. 2010. Toxic effect of heavy metals on aquatic environment. Int. J. Biol. Chem. Sci., 4(4): 939-952.

DOI: http://dx.doi.org/10.4314/ijbcs.v4i4.62976

Berrada S, Squalli FZ, Squalli HT, Hannin M, El Oualti A, El Ouali Lalami A. 2014. Recyclage des effluents du service d'hémodialyse de l'hôpital Al Ghassani de la ville de Fès: caractérisation avant et après traitement. J. Mater. Environ. Sci., 5: 22652277.

Bouzid J, Chahlaoui A, Zaid A, My Mehdi R, Bouhlou L. 2013. Etude bactériologique et physicochimique des effluents liquides de l'hôpital Mohamed V de Meknès. Science Lib, 5(130803): 1-15.

Carraro E. Bonetta S. Bertino C. Lorenzi E. Bonetta S. Gilli G. 2016. Hospital effluents management: Chemical, physical, microbiological risks and legislation in different countries. Journal of Environmental Management, 168: 185$199 . \quad$ DOI: http://doi.org/10.1016/j.jenvman.2015.11.0 21

Chiffoleau JC. 2001. La contamination métallique. Rapport technique, programme scientifique Seine-Aval. IFREMER: Région Haute Normandie. p.6-7.

Chonova T, Keck F, Labanowski J, Montuelle B, Rimet F, Bouchez A. 2016. Separate treatment of hospital and urban wastewaters: a real scale comparison of effluents and their effect on microbial communities. Sci. Tota. Environ., 542: 965-975.

DOI : https://doi.org/10.1016/j.scitotenv.2015.10 .161

Evens E. 2004. Evaluation des risques sanitaires et écotoxicologiques liés aux effluents hospitaliers. Thèse de doctorat, Institut national des sciences appliquées de Lyon, Lyon, p. 259.

El-Ogri F, Ouazzani N, Boraam F, Mandi L. 2016. A survey of wastewaters generated by a hospital in Marrakech city and their characterization. Desalin Water Treat, 57: 17061-17074. DOI: 10.1080/19443994.2016.1138328

El Mehdi T, Lahcen B, Chakib N, Kawtar FB. 2009. Caractérisation des effluents liquides de l'hôpital Al Ghassani, CHU Hassan II de Fès, Maroc. Les effluents liquides des établissements de santé : état des lieux et perspectives de gestion. Rev Hospi France, $714: 47-50$.

El Morhit M, Fekhaoui M, Serghini A, El Blidi, S. El Abidi A, Yahyaoui A, Hachimi M. 2012. Étude de l'évolution spatiotemporelle des paramètres hydrologiques caractérisant la qualité des eaux de l'estuaire du Loukkos (Maroc). Bulletin de l'Institut Scientifique, section Sciences de la Vie, 34(2) : 151-162.

EL Mountassir R, Bennani B, Merzouki H, Benjelloun Touimi G, Boumchita S, Benjelloun Y, Lahrichi A. 2017. Characterization of the chemical and bacteriological risks of the effluents from some services of the Hassan II Hospital Center in Fez. JMES, 8(7): 2288-2295.

Fekadu S, Merid Y, Beyene H, Teshome W, Gebre-Selassie S. 2015. Assessment of antibioticand disinfectant-resistant bacteria in hospital wastewater, South Ethiopia: a cross-sectional study. J Infect Dev Ctries, 9(2): 149-156.

DOI: https://doi.org/10.3855/jidc.4808

Hamaidi MS, Hamaidi F, Zoubiri A, Benouaklil F, Dhan Y. 2009. Etude de la dynamique des populations phytoplanctoniques et résultats préliminaires sur les blooms toxiques a cyanobacteries dans le barrage de Ghrib (Ain Defla-Algérie). European Journal of Scientific Research, 32(3): 369-380.

Ike CC, Akwari DK. 2017. Microbiological and physicochemical quality of hospital "X" effluent in Aba, Abia State, Nigeria. IJRDO J Applied Sci, 3(5): 26-37.

Lim SR, Schoenung JM. 2010. Human health and ecological toxicity potentials due to heavy metal content in waste electronic devices with flat panel displays. $J$ Hazardous Materials, 177: 251-259. DOI: 
https://doi.org/10.1016/j.jhazmat.2009.12. 025

Maheshwari M, Yaser NH, Naz S, Fatima M, Ahmad I. 2016. Emergence of ciprofloxacinresistant extended-spectrum $\beta$-lactamase-producing enteric bacteria in hospital wastewater and clinical sources. $J$ Glob Antimicrob Resist, 5: 22-25. DOI: 10.1016/j.jgar.2016.01.008

Oliveira H, Lopes T, Almeida T, Pereira ML, Santos C. 2012. Cadmium-induced genetic instability in mice testis. Hum Exp Toxicol, 31(12): 1228-1236.

DOI:

https://doi.org/10.1177\%2F096032711244 5937

Prozialeck WC, Edwards JR. 2012. Mechanisms of Cadmium-Induced Proximal Tubule Injury: New Insights with Implications for Biomonitoring and Therapeutic Interventions. J Pharmacol Exp Ther, 343: 2-12. DOI : https://doi.org/10.1124/jpet.110.166769

Nations Unies. 2017. Rapport mondial sur la mise en valeur des ressources en eau. Les eaux usées- Une ressource inexploitée. UNESCO : Paris.

République du Bénin. 2001. Décret $n^{\circ} 2001$ 109 du 4 avril 2001 fixant les normes de qualité des eaux résiduaires en république du Bénin. Journal Officiel de la République du Bénin. 1- 27.

Rodier J, Legube B, Merlet N, Brunet R. 2009. L'Analyse de l'Eau (9 edn). Dunod : Paris.

Sadek S, Elkharrim K, Khadmaoui A, Guissouss M, Benelharkati F, Elmarkhi M,
Driss B. 2012. Caractérisation physicochimique des eaux usées de l'hôpital provincial de Sidi Kacem (Maroc). Science Lib., 4(120713) : 1-8.

Santos LH, Gros M, Rodriguez-Mozaz S, Delerue-Matos C, Pena A, Barcelo D, Montenegro MC. 2013. Contribution of hospital effluents to the load of pharmaceuticals in urban wastewaters: identification of ecologically relevant pharmaceuticals. Sci. Total. Environ., 461: 302-316.

DOI: https://doi.org/10.1016/j.scitotenv.2013.04 .077

Verlicchi P, Al Aukidy M, Galletti A, Petrovic M, Barcelo D. 2012. Hospital effluent: investigation of the concentrations and distribution of pharmaceuticals and environmental risk assessment. Sci. Total Environ., 430: 109-118. DOI: https://doi.org/10.1016/j.scitotenv.2012.04 .055

Verlicchi P, Galletti A, Petrovic M, Barcelo D. 2010. Hospital effluents as a source of emerging pollutants: an overview of micropollutants and sustainable treatment options. J Hydrol, 389: 416-428. DOI: 10.1016/j.jhydrol.2010.06.005

Word Health Organization. 1999. Safe Management of Wastes from Health-care Activities. WHO: Geneva.

Word Health Organization. 2014. Safe Management of Wastes from Health-care Activities (2nd edn). WHO: Geneva. 